US-FT/7-94

hep-th/9405151

May, 1994

\title{
TYPE B TOPOLOGICAL MATTER, KODAIRA-SPENCER THEORY, AND MIRROR SYMMETRY
}

\author{
J.M.F. LABASTIDA AND M. MARIÑNO \\ Departamento de Física de Partículas \\ Universidade de Santiago \\ E-15706 Santiago de Compostela, Spain
}

\begin{abstract}
Perturbing usual type B topological matter with vector $(0,1)$-forms we find a topological theory which contains explicitly Kodaira-Spencer deformation theory. It is shown that, in genus zero, three-point correlation functions give the Yukawa couplings for a generic point in the moduli space of complex structures. This generalization of type B topological matter seems to be the correct framework to understand mirror symmetry in terms of two-dimensional topological field theories.
\end{abstract}

\footnotetext{
^ E-mail: LABASTIDA@GAES.USC.ES
} 
Topological matter in two dimensions $[1,2,3,4]$ has become a very interesting framework $[5,6]$ to understand mirror symmetry [7-13] in Calabi-Yau manifolds. Type A topological models are topological sigma models whose observables depend on the moduli space of Kähler forms on the target space but are independent of the complex structure $[5,14]$. Type B topological models are also topological sigma models but in this case the observables depend on the complex structure and are independent of the Kähler form [5]. Mirror symmetry is realized in this context by stating that the observables of type A models for a manifold are related to the observables of type B models for its mirror manifold. While the dependence on the moduli space of Kähler forms in type A models is well established [5,14], the dependence on the moduli space of complex structures has not yet been developed for type $\mathrm{B}$ models. This is because type B models are defined $[5,4]$ just at one point on moduli space. The aim of this paper is to present a generalization of type B models whose observables depend on the moduli space of complex structures. As it will be shown below, this work reveals a clear parallelism between the structures of type A and type B topological matter.

Before describing the construction of the new form of type B topological matter let us briefly review some results on the theory of deformations of complex structures that will be needed in what follows. A standard reference on this topic is [15]. A complex manifold $M$ can be considered as a set of domains $\left\{\mathcal{U}_{j}\right\}$ in $\mathbf{C}^{n}$ glued by coordinate transformations $f_{j k}\left(z_{k}\right)$ which are biholomorphic functions. A deformation of the complex structure of $M$ is a variation of these transition functions depending on some complex parameters $t_{1}, \cdots, t_{s}$. This deformation generates a family of complex manifolds that will be denoted by $M_{t}$. It follows from this definition that infinitesimal deformations of $M$ are always elements in the sheaf cohomology group $H^{1}(M, T M)$. However, the converse is not true. In other words, topological obstructions to integrate an infinitesimal deformation are found in general, and therefore not every $\bar{\partial}$-closed vector $(0,1)$-form is associated to a family of complex manifolds. Alternatively, a deformation of the complex structure of $M$ can be represented by a vector $(0,1)$-form $\phi(t)$ verifying the Kodaira-Spencer 
equation:

$$
\bar{\partial} \phi(t)=\frac{1}{2}[\phi(t), \phi(t)]
$$

and the initial condition $\phi(0)=0$. In (1) the bracket between a vector $(0, p)$-form $\phi=\sum_{\alpha} \phi^{\alpha} \partial_{\alpha}$ and a vector $(0, q)$-form $\psi=\sum_{\alpha} \psi^{\alpha} \partial_{\alpha}$ is defined by:

$$
[\phi, \psi]=\sum_{\alpha, \beta}\left(\phi^{\alpha} \wedge \partial_{\alpha} \psi^{\beta}-(-1)^{p q} \psi^{\alpha} \wedge \partial_{\alpha} \phi^{\beta}\right) \partial_{\beta}
$$

In this framework, the problem of topological obstructions can be formulated as follows: one can show that every $\phi(t)$ representing a complex deformation verifies $\left(\frac{\partial \phi(t)}{\partial t}\right)_{t=0} \in H^{1}(M, T M)$, so an infinitesimal deformation $\phi_{1}$ is unobstructed if one can find a solution $\phi(t)$ to $(1)$ such that that $\left(\frac{\partial \phi(t)}{\partial t}\right)_{t=0}=\phi_{1}$. It has been proven by Tian [16] and Todorov [17] that, when $M$ is a Calabi-Yau manifold, every infinitesimal deformation is unobstructed. More precisely, given $\phi_{1} \in H^{1}(M, T M)$, there is a power expansion in a parameter $t$ (which can be understood as a parameter in the moduli space of complex structures):

$$
\phi(t)=\phi_{1} t+\phi_{2} t^{2}+\cdots
$$

such that $\phi(t)$ satisfies (1) and therefore corresponds to a deformation of the complex structure. In fact, the vector $(0,1)$-forms appearing in this series are obtained by solving inductively the Kodaira-Spencer equation at order $n$ in $t$ :

$$
\bar{\partial} \phi_{n}=\frac{1}{2} \sum_{i=1}^{n-1}\left[\phi_{i}, \phi_{n-i}\right]
$$

In this way, when $M$ is a Calabi-Yau, deformations of the complex structure are in one to one correspondence with $\bar{\partial}$-closed vector $(0,1)$-forms.

In this paper we will construct a generalization of type B topological matter which explicitly contains Kodaira-Spencer deformation theory and, in some way, 
also Tian's results. Let us first present a brief account of the original model. There are two equivalent ways two obtain the two types, $\mathrm{A}$ and $\mathrm{B}$, of topological matter by twisting $N=2$ supersymmetry. One consists of taking an $N=2$ chiral multiplet and twist with each of the two $U(1)$ chiral currents [5]. The other one consists of twisting by only one of the $U(1)$ chiral currents both, the $N=2$ chiral multiplet and the $N=2$ twisted chiral multiplet [4]. In either case we will be considering the B model. The target space $M$ must be a Calabi-Yau manifold in order to avoid anomalies [5]. The field content of this model consists of a set of local coordinates, $x^{I}$ and $x^{\bar{I}}$, which describe a map from a Riemann surface $\Sigma$ to $M$, anticommuting fields $\eta^{\bar{I}}, \theta_{I}$ and $\rho_{\mu}^{I}$, and auxiliary fields $F^{I}$ and $F^{\bar{I}}$. We follow mainly the notation in [4] after making the field redefinition of the original fields $\chi^{\bar{I}}$ and $\bar{\chi}^{\bar{I}}$ suggested in [5]:

$$
\begin{aligned}
\eta^{\bar{I}} & =\chi^{\bar{I}}+\bar{\chi}^{\bar{I}}, \\
\theta_{I} & =g_{I \bar{J}}\left(\chi^{\bar{J}}-\bar{\chi}^{\bar{J}}\right),
\end{aligned}
$$

being $g_{I \bar{J}}$ a metric on $M$. The model possesses the following topological $Q-$ symmetry:

$$
\begin{aligned}
{\left[Q, x^{I}\right] } & =0, & & \left\{Q, \eta^{\bar{I}}\right\}=0, \\
{\left[Q, x^{\bar{I}}\right] } & =\eta^{\bar{I}}, & & \left\{Q, \theta_{I}\right\}=g_{I \bar{J}} F^{\bar{J}}, \\
\left\{Q, \rho_{z}^{I}\right\} & =\partial_{z} x^{I}, & & {\left[Q, F^{I}\right]=D_{z} \rho_{\bar{z}}^{I}-D_{\bar{z}} \rho_{z}^{I}+R_{J \bar{L} K}^{I} \eta^{\bar{L}} \rho_{z}^{J} \rho_{\bar{z}}^{K}, } \\
\left\{Q, \rho_{\bar{z}}^{I}\right\} & =\partial_{\bar{z}} x^{I}, & & {\left[Q, F^{\bar{I}}\right]=-\Gamma_{\bar{J} \bar{K}}^{\bar{I}} \eta^{\bar{J}} F^{\bar{K}}, }
\end{aligned}
$$

whose operator satisfies $Q^{2}=0$. The action of the type B model takes the form:

$$
\begin{aligned}
& S=\int_{\Sigma} d^{2} z\left[g_{I \bar{J}}\left(\partial_{z} x^{I} \partial_{\bar{z}} x^{\bar{J}}+\partial_{\bar{z}} x^{I} \partial_{z} x^{\bar{J}}\right)-\rho_{z}^{I}\left(g_{I \bar{J}} D_{\bar{z}} \eta^{\bar{J}}+D_{\bar{z}} \theta_{I}\right)\right. \\
&\left.-\rho_{\bar{z}}^{I}\left(g_{I \bar{J}} D_{z} \eta^{\bar{J}}-D_{z} \theta_{I}\right)-R_{J \bar{L} K}^{I} \eta^{\bar{L}} \rho_{z}^{J} \rho_{\bar{z}}^{K} \theta_{I}-g_{I \bar{J}} F^{I} F^{\bar{J}}\right]
\end{aligned}
$$

which is easily seen to be $Q$-exact:

$$
S=\left\{Q, \int_{\Sigma} d^{2} z\left[g_{I \bar{J}}\left(\rho_{z}^{I} \partial_{\bar{z}} x^{\bar{J}}+\rho_{\bar{z}}^{I} \partial_{z} x^{\bar{J}}\right)-F^{I} \theta_{I}\right]\right\} .
$$


This last relation implies that the model under consideration possesses two of the most common features of topological quantum field theories. On one hand, the energy-momentum tensor is $Q$-exact. On the other hand, the partition function and the vacuum expectation value of products of $Q$-invariant operators can be evaluated in the weak coupling regime. This last observation implies that the observables of the theory do not depend on the Kähler class chosen on $M$. Notice however that, as pointed out in [5], the type B model depends on the complex structure of the target manifold $M$ through the $Q$-transformation, which depends crucially on the assignation of holomorphic and antiholomorphic coordinates. The model is defined only for a particular point in the moduli space of complex structures, corresponding to the Calabi-Yau manifold $M$ which we have chosen as base point. The situation in type A topological matter is quite different: there the action can be written as a $Q$ exact term plus a topological term, involving only the pullback of the Kähler class. This remaining piece allows one to include a explicit parametrization of the moduli space of Kähler forms (as one can see, for example, in [14]). The $Q$-symmetry for type A models does not depend on the complex structure and therefore observables are functions on the moduli space of Kähler forms. It is difficult to exploit mirror symmetry by means of topological matter using as the partner of the type A model the usual type B model, because moduli space structures seems to be essential to mirror symmetry as well as to mirror computations $[18,12,11]$. As suggested in [5], mirror symmetry should be better understood in the framework of topological field theory if one considers not only the original lagrangian, but the topological family obtained adding perturbations to it. In this way, the generic lagrangian corresponding to the topological family should depend on a set of parameters related, if we take the appropriate perturbation, to the moduli space of complex structures.

The standard procedure for perturbing topological theories is the use of descent equations $[1,5,4]$. As advocated in [4], a very useful tool in generating the fields entering descent equations is the vector operator $G_{\mu} . N=2$ supersymmetry in two dimensions possesses four spinor generators. After the twisting, two of these 
generators lead to two scalar operators, being $Q$ one of them, and a vector operator $G_{\mu}$. For twisted models to have the symmetry generated by $G_{\mu}$ the existence of covariantly constant vectors on $\Sigma$ is required. This requirement does not hold in general and therefore typically the $G_{\mu}$ symmetry is disregarded. However, this symmetry is useful for two reasons. First, as shown in [4], its gauging leads to the coupling of topological matter to topological gravity. Second, it generates operators which satisfy the descent equations. It is in this last respect that it will be used in this work.

To understand why the operator $G_{\mu}$ generates solutions to the descent equation let us recall that the topological algebra [4] identifies it as the $Q$-partner of the momentum operator:

$$
\left\{Q, G_{\mu}\right\}=P_{\mu} .
$$

Starting with a field $\phi^{(0)}(x)$ which satisfies,

$$
\left[Q, \phi^{(0)}(x)\right]=0,
$$

one can construct other fields using the operators $G_{\mu}$. These fields, which we will call partners or descendants, are antisymmetric tensors defined as,

$$
\begin{array}{r}
\phi_{\mu}^{(1)}(x)=\left[G_{\mu}, \phi^{(0)}(x)\right\}, \\
\phi_{\mu \nu}^{(2)}(x)=\left[G_{\mu},\left[G_{\nu}, \phi(x)\right\}\right\} .
\end{array}
$$

Relation (9) guarantees that these operators satisfy the topological descent equations,

$$
d \phi^{(n)}=\left[Q, \phi^{(n+1)}\right\},
$$

which in turn imply that quantities like,

$$
W_{\phi}^{\Sigma}=\int_{\Sigma} \phi^{(2)},
$$

are $Q$-invariant. Operators of the form (13) are very useful because they can 
be added to the lagrangian to build families of topological models. This is the procedure that will be used to construct the perturbed B model.

The $G_{\mu}$-transformations of the fields can be read from [4]. They take the form:

$$
\begin{aligned}
{\left[G_{z}, x^{I}\right] } & =\rho_{z}^{I}, & & \left\{G_{z}, \eta^{\bar{I}}\right\}=\partial_{z} x^{\bar{I}}, \\
{\left[G_{\bar{z}}, x^{I}\right] } & =\rho_{\bar{z}}^{I}, & & \left\{G_{\bar{z}}, \eta^{\bar{I}}\right\}=\partial_{\bar{z}} x^{\bar{I}}, \\
{\left[G_{z}, x^{\bar{I}}\right] } & =0, & & \left\{G_{z}, \theta_{I}\right\}=g_{I \bar{J}} \partial_{z} x^{\bar{J}}+\Gamma_{I K}^{J} \rho_{z}^{K} \theta_{J}, \\
{\left[G_{\bar{z}}, x^{\bar{I}}\right] } & =0, & & \left\{G_{\bar{z}}, \theta_{I}\right\}=g_{I \bar{J}} \partial_{\bar{z}} x^{\bar{J}}+\Gamma_{I K}^{J} \rho_{\bar{z}}^{K} \theta_{J}, \\
\left\{G_{z}, \rho_{z}^{I}\right\} & =0, & {\left[G_{z}, F^{I}\right] } & =-\Gamma_{J K}^{I} \rho_{z}^{J} F^{K}, \\
\left\{G_{\bar{z}}, \rho_{z}^{I}\right\} & =-F^{I}+\Gamma_{J K}^{I} \rho_{z}^{J} \rho_{\bar{z}}^{K}, & & {\left[G_{\bar{z}}, F^{I}\right]=\Gamma_{J K}^{I} \rho_{\bar{z}}^{J} F^{K}, } \\
\left\{G_{z}, \rho_{\bar{z}}^{I}\right\} & =F^{I}-\Gamma_{J K}^{I} \rho_{z}^{J} \rho_{\bar{z}}^{K}, & & {\left[G_{z}, F^{\bar{I}}\right]=-D_{z}\left(\eta^{\bar{I}}-g^{\bar{I} J} \theta_{J}\right)+R_{L \bar{K}}^{\bar{I} J} \rho_{z}^{L} \eta^{\bar{K}} \theta_{J}, } \\
\left\{G_{\bar{z}}, \rho_{\bar{z}}^{I}\right\} & =0, & {\left[G_{\bar{z}}, F^{\bar{I}}\right] } & =D_{z}\left(\eta^{\bar{I}}+g^{\bar{I} J} \theta_{J}\right)+R_{L \bar{K}}^{\bar{I} J} \rho_{\bar{z}}^{L} \eta^{\bar{K}} \theta_{J} .
\end{aligned}
$$

The observables of type B topological matter have the form:

$$
\phi^{(0)}=A_{\bar{I}_{1} \cdots \bar{I}_{p}}{ }^{J_{1} \cdots J_{q}} \eta^{\bar{I}_{1}} \cdots \eta^{\bar{I}_{p}} \theta_{J_{1}} \cdots \theta_{J_{q}}
$$

where $A$ is an element in $H^{p}\left(M, \wedge^{q} T M\right)$. Notice that these are observables only on-shell, i.e., taking into account the field equations, because the action of $Q$ on $\theta_{I}$ gives terms proportional to $\frac{\delta S}{\delta F^{j}}$. As we mentioned above, Kodaira-Spencer theory and Tian's results imply that closed vector $(0,1)$-forms are in one to one correspondence with deformations of the complex structure, so we expect that the appropriate topological family will be obtained perturbing with observables of the form:

$$
\phi^{(0)}=A_{\bar{I}}^{J} \eta^{\bar{I}} \theta_{J}
$$

where $\partial_{[\bar{K}} A_{\bar{I}]}^{J}=0$. In this case one finds:

$$
\left[Q, \phi^{(0)}\right]=A_{\bar{I}}^{J} \eta^{\bar{I}} \frac{\delta S}{\delta F^{J}} .
$$

The fact that $\phi^{(0)}$ is $Q$-closed only on-shell has important consequences [5]: now the topological descent equations are true only modulo terms proportional to the field 
equations, and this implies in turn that we must change the topological operator $Q$ if we want the perturbed lagrangian to be $Q$-closed. To obtain the form of the descent equations, including the additional terms, we will use the operator $G_{\mu}$ introduced above. Taking into account (9), one finds that the operators $\phi_{\mu}^{(1)}$ and $\phi_{\mu \nu}^{(2)}$ defined in terms of an operator $\phi^{(0)}$ as in (11) satisfy the following modified descent equations:

$$
\begin{aligned}
{\left[Q, \phi_{\mu}^{(1)}\right\} } & =\left[P_{\mu}, \phi^{(0)}\right]+\left[G_{\mu},\left[Q, \phi^{(0)}\right\}\right\} \\
{\left[Q, \phi_{\mu \nu}^{(2)}\right\} } & =\left[P_{\mu}, \phi_{\nu}^{(1)}\right]-\left[P_{\nu}, \phi_{\mu}^{(1)}\right]+\left[G_{\mu},\left[G_{\nu},\left[Q, \phi^{(0)}\right\}\right\}\right\} .
\end{aligned}
$$

For an operator like (16) satisfying (17) the last terms of these equations represent additional contributions to the standard topological descent equations (12). These extra terms are proportional to field equations due to (17) and the fact that the action (7) is $G_{\mu}$ invariant. Actually, the simplest way to compute the form of these extra terms is to us this invariance of the action (7). Let us denote generically by $\psi_{i}$ the fields in the theory, and by $\delta S$ the variation of the action due to an arbitrary variation $\delta \psi_{i}$ of the fields. The invariance of the action (7) under $G_{\mu}$ implies:

$$
\left[G_{\mu}, \delta S\right]=\left[G_{\mu}, \sum_{i} \frac{\delta S}{\delta \psi_{i}}\right\} \delta \psi_{i}+\sum_{i} \frac{\delta S}{\delta \psi_{i}}\left[G_{\mu}, \delta \psi_{i}\right\}=0
$$

The useful consequence of this relation is that the transformation under $G_{\mu}$ of the field equations can be read easily after using (14).

To build the perturbation of the action (7) notice that since the second term of the second equation in (18) is linear in the field equations, i.e.,

$$
\left[Q, \phi^{(2)}\right\}=d \phi^{(1)}+\sum_{i} \frac{\delta S}{\delta \psi_{i}} \zeta_{i}
$$

where $\zeta_{i}$ are quantities to be determined, the generalized action,

$$
S(t)=S+t \int_{\Sigma} \phi^{(2)},
$$


is invariant up to terms of order $t^{2}$ under the new topological symmetry,

$$
\left[Q_{t}, \psi_{i}\right]=\left[Q, \psi_{i}\right]-t \zeta_{i}
$$

Let us study the perturbation associated to a closed vector $(0,1)$-form as in (16). Using (11) one finds that the descendants are:

$$
\begin{aligned}
\phi_{z}^{(1)} & =D_{K} A_{\bar{I}}^{J} \rho_{z}^{K} \eta^{I} \theta_{J}+A_{\bar{I}}^{J}\left(\partial_{z} x^{\bar{I}} \theta_{J}+g_{J \bar{K}} \partial_{z} x^{\bar{K}} \eta^{\bar{I}}\right), \\
\phi_{z \bar{z}}^{(2)} & =D_{L} D_{K} A_{\bar{I}}^{J} \eta^{\bar{I}} \rho_{z}^{L} \rho_{\bar{z}}^{K} \theta_{J}+D_{K} A_{\bar{I}}^{J}\left(\rho_{z}^{K} \partial_{\bar{z}} x^{\bar{I}}-\rho_{\bar{z}}^{K} \partial_{z} x^{\bar{I}}\right) \theta_{J} \\
& +D_{K} A_{\bar{I}}^{J}\left(\rho_{z}^{K} \partial_{\bar{z}} x^{\bar{L}}-\rho_{\bar{z}}^{K} \partial_{z} x^{\bar{L}}\right) g_{J \bar{L}} \eta^{\bar{I}}+g_{J \bar{K}} A_{\bar{I}}^{J}\left(\partial_{z} x^{\bar{I}} \partial_{\bar{z}} x^{\bar{K}}+\partial_{\bar{z}} x^{\bar{I}} \partial_{z} x^{\bar{K}}\right) \\
& +D_{K} A_{\bar{I}}^{J} F^{K} \eta^{\bar{I}} \theta_{J} .
\end{aligned}
$$

To obtain the quantities $\zeta_{i}$ in (20) we use (19) for the field equations needed. Of course, this could be obtained acting directly with $G_{\mu}$ on the field equations. However, the use of (19) simplifies notably the computations. One obtains,

$$
\begin{aligned}
\left\{G_{\bar{z}}, \frac{\delta S}{\delta F^{I}}\right\} & =\frac{\delta S}{\delta \rho_{z}^{I}}-\Gamma_{I J}^{K} \rho_{\bar{z}}^{J} \frac{\delta S}{\delta F^{K}}, \\
\left\{G_{z}, \frac{\delta S}{\delta F^{I}}\right\} & =-\frac{\delta S}{\delta \rho_{\bar{z}}^{I}}+\Gamma_{I J}^{K} \rho_{z}^{J} \frac{\delta S}{\delta F^{K}}, \\
\left\{G_{z}, \frac{\delta S}{\delta \rho_{z}^{I}}\right\} & =\frac{\delta S}{\delta x^{I}}-\Gamma_{I K}^{J} \rho_{\bar{z}}^{K} \frac{\delta S}{\delta \rho_{\bar{z}}^{J}}+\Gamma_{I J}^{K} \theta_{K} \frac{\delta S}{\delta \theta_{J}}-\Gamma_{I K}^{J} F^{K} \frac{\delta S}{\delta F^{J}}+R^{\bar{L} J}{ }_{I \bar{K}} \eta^{\bar{K}} \theta_{J} \frac{\delta S}{\delta F^{\bar{L}}},
\end{aligned}
$$

and, finally, the quantities $\zeta_{i}$ are read off from (20):

$$
\begin{aligned}
\zeta_{x^{J}} & =A_{\bar{I}}^{J} \eta^{\bar{I}}, \\
\zeta_{\theta_{J}} & =-A_{\bar{I}}^{K} \eta^{\bar{I}} \Gamma_{J K}^{L} \theta_{L}, \\
\zeta_{\rho_{z}^{J}} & =-\partial_{K} A_{\bar{I}}^{J} \rho_{z}^{K} \eta^{\bar{I}}-A_{\bar{I}}^{J} \partial_{z} x^{\bar{I}}, \\
\zeta_{\rho_{\bar{z}}^{J}} & =-\partial_{K} A_{\bar{I}}^{J} \rho_{\bar{z}}^{K} \eta^{\bar{I}}-A_{\bar{I}}^{J} \partial_{\bar{z}} x^{\bar{I}}, \\
\zeta_{F^{J}} & =D_{L} D_{K} A_{\bar{I}}^{J} \eta^{\bar{I}} \rho_{z}^{L} \rho_{\bar{z}}^{K}+D_{K} A_{\bar{I}}^{J}\left(\rho_{z}^{K} \partial_{\bar{z}} x^{\bar{I}}-\rho_{\bar{z}}^{K} \partial_{z} x^{\bar{I}}\right)+\partial_{K} A_{\bar{I}}^{J} F^{K} \eta^{\bar{I}}, \\
\zeta_{F^{\bar{J}}} & =A_{\bar{I}}^{M} R_{M \bar{K}}^{\bar{J} L} \eta^{\bar{I}} \eta^{\bar{K}} \theta_{L},
\end{aligned}
$$

while all the rest vanish. 
As discussed above, adding $-t \zeta_{i}$ to the old $Q$ one obtains a new one which only works at order $t$. It is natural to ask under what conditions we can construct a topological symmetry $Q^{\prime}$ verifying $Q^{\prime 2}=0$ at every order in $t$. The previous construction suggests to take:

$$
\begin{aligned}
{\left[Q^{\prime}, x^{J}\right] } & =-A_{\bar{I}}^{J} \eta^{\bar{I}}, \\
{\left[Q^{\prime}, x^{\bar{J}}\right] } & =\eta^{\bar{J}} \\
\left\{Q^{\prime}, \rho_{z}^{J}\right\} & =\partial_{z} x^{J}+\partial_{K} A_{\bar{I}}^{J} \rho_{z}^{K} \eta^{\bar{I}}+A_{\bar{I}}^{J} \partial_{z} x^{\bar{I}}=\partial_{z} x^{J}+\left\{G_{z}, A_{\bar{I}}^{J} \eta^{\bar{I}}\right\} \\
\left\{Q^{\prime}, \rho_{\bar{z}}^{J}\right\} & =\partial_{\bar{z}} x^{J}+\partial_{K} A_{\bar{I}}^{J} \rho_{\bar{z}}^{K} \eta^{\bar{I}}+A_{\bar{I}}^{J} \partial_{\bar{z}} x^{\bar{I}}=\partial_{\bar{z}} x^{J}+\left\{G_{\bar{z}}, A_{\bar{I}}^{J} \eta^{\bar{I}}\right\}, \\
\left\{Q^{\prime}, \eta^{\bar{J}}\right\} & =0, \\
\left\{Q^{\prime}, \theta_{I}\right\} & =g_{I \bar{J}} F^{\bar{J}}+\partial_{I} A_{\bar{K}}^{J} \eta^{\bar{K}} \theta_{J}, \\
{\left[Q^{\prime}, F^{I}\right] } & =D_{z} \rho_{\bar{z}}^{I}-D_{\bar{z}} \rho_{z}^{I}+R^{I}{ }_{J \bar{L} K} \eta^{\bar{L}} \rho_{z}^{J} \rho_{\bar{z}}^{K}-D_{L} D_{K} A_{\bar{J}}{ }^{I} \eta^{\bar{J}} \rho_{z}^{L} \rho_{\bar{z}}^{K} \\
& -D_{K} A_{\bar{J}}^{I}\left(\rho_{z}^{K} \partial_{\bar{z}} x^{\bar{J}}+\rho_{\bar{z}}^{K} \partial_{z} x^{\bar{J}}\right)-\partial_{K} A_{\bar{J}}^{I} F^{K} \eta^{\bar{J}} \\
{\left[Q^{\prime}, F^{\bar{J}}\right] } & =-F^{\bar{L}} \eta^{\bar{K}}\left(\Gamma_{\bar{L} \bar{K}}^{\bar{J}}-g^{\bar{J} I} g_{\bar{L} M} D_{I} A_{\bar{K}}^{M}\right)
\end{aligned}
$$

where the field $F^{\bar{J}}$ has been redefined in such a way that the terms involving auxiliary fields in the final action reduce to the quadratic form $g_{I \bar{J}} F^{I} F^{\bar{J}}$ :

$$
F^{\bar{J}} \rightarrow F^{\bar{J}}+g^{\bar{J} I} D_{I} A_{\bar{K}}^{J} \eta^{\bar{K}} \theta_{J}
$$

Imposing $\left\{Q^{\prime},\left[Q^{\prime}, x^{J}\right]\right\}=0$ gives the following constraint on $A_{\bar{I}}{ }^{J}$ :

$$
\partial_{\bar{K}} A_{\bar{I}}^{J} \eta^{\bar{K}} \eta^{\bar{I}}-A_{\bar{L}}^{K} \partial_{K} A_{\bar{I}}^{J} \eta^{\bar{L}} \eta^{\bar{I}}=0
$$

This is just the Kodaira-Spencer equation (1) for the vector $(0,1)$-form $A_{\bar{I}}^{J}$. Surprisingly enough, this is a necessary and sufficient condition for $Q^{\prime}$ to be a nilpotent operator. To see this notice first that the operators $G_{\mu}$ still close a topological algebra with $Q^{\prime}$, i.e., $\left\{Q^{\prime}, G_{\mu}\right\}=P_{\mu}$. Using this fact it is easy to prove nilpotency 
of $Q^{\prime}$ on $\rho_{\mu}^{I}$. For $F^{J}$, computations are in principle more involved, but the field,

$$
\tilde{F}^{I}=F^{I}-\Gamma_{J K}^{I} \rho_{z}^{J} \rho_{\bar{z}}^{K}
$$

has the simple transformation,

$$
\left[Q^{\prime}, \tilde{F}^{I}\right]=\partial_{z} \rho_{\bar{z}}^{I}-\partial_{\bar{z}} \rho_{z}^{I}-\left[G_{z},\left\{G_{\bar{z}}, A_{\bar{J}}^{I} \eta^{\bar{J}}\right\}\right]
$$

and, again the nilpotency of $Q^{\prime}$ on $\tilde{F}^{J}$ can be easily proved using the topological algebra. Finally, for the field $F^{\bar{J}}$ an explicit computation leads also to the same conclusion.

Given a vector $(0,1)$-form which satisfies the Kodaira-Spencer equation we have at our disposal a nilpotent operator $Q^{\prime}$ which satisfies the topological algebra and reduce to the original one setting the vector $(0,1)$-form $A_{\bar{I}}^{J}$ to zero. Having an off-shell nilpotent operator like $Q^{\prime}$, the simplest way to construct a $Q^{\prime}$-invariant action is to take one which is a $Q^{\prime}$-transformation. We will define the modified action as in (8), but using now $Q^{\prime}$ instead of $Q$. The new action takes the form:

$$
S^{\prime}=\left\{Q^{\prime}, \int_{\Sigma} d^{2} z\left[g_{I \bar{J}}\left(\rho_{z}^{I} \partial_{\bar{z}} x^{\bar{J}}+\rho_{\bar{z}}^{I} \partial_{z} x^{\bar{J}}\right)-F^{I} \theta_{I}\right]\right\}=S+\int_{\Sigma} \phi^{(2)},
$$

where $\phi^{(2)}$ is given as in (23), with the only difference that now $A_{\bar{I}}{ }^{J}$ verifies the Kodaira-Spencer equation instead of being closed. The explicit form of the new lagrangian is:

$$
\begin{aligned}
\mathcal{L}= & g_{I \bar{J}}\left(\partial_{z} x^{I} \partial_{\bar{z}} x^{\bar{J}}+\partial_{\bar{z}} x^{I} \partial_{z} x^{\bar{J}}\right)-\rho_{z}^{I}\left(g_{I \bar{J}} D_{\bar{z}} \eta^{\bar{J}}+D_{\bar{z}} \theta_{I}\right) \\
& -\rho_{\bar{z}}^{I}\left(g_{I \bar{J}} D_{z} \eta^{\bar{J}}-D_{z} \theta_{I}\right)-R_{J \bar{L} K}^{I} \eta^{\bar{L}} \rho_{z}^{J} \rho_{\bar{z}}^{K} \theta_{I}-g_{I \bar{J}} F^{I} F^{\bar{J}} \\
& +D_{L} D_{K} A_{\bar{I}}^{J} \eta^{\bar{I}} \rho_{z}^{L} \rho_{\bar{z}}^{K} \theta_{J}+D_{K} A_{\bar{I}}^{J}\left(\rho_{z}^{K} \partial_{\bar{z}} x^{\bar{I}}-\rho_{\bar{z}}^{K} \partial_{z} x^{\bar{I}}\right) \theta_{J} \\
& +D_{K} A_{\bar{I}}^{J}\left(\rho_{z}^{K} \partial_{\bar{z}} x^{\bar{L}}-\rho_{\bar{z}}^{K} \partial_{z} x^{\bar{L}}\right) g_{J \bar{L}} \eta^{\bar{I}}+g_{J \bar{K}} A_{\bar{I}}^{J}\left(\partial_{z} x^{\bar{I}} \partial_{\bar{z}} x^{\bar{K}}+\partial_{\bar{z}} x^{\bar{I}} \partial_{z} x^{\bar{K}}\right) .
\end{aligned}
$$

At this point one can ask what is the relation of this theory to the one we constructed previously along the suggestions given in [5]. Recall that, according 
to Tian's theorem, when $M$ is a Calabi-Yau manifold there is a one to one correspondence between closed vector $(0,1)$-forms and deformations of the complex structure: the series (3) associates to every $\phi_{1}$ verifying $\bar{\partial} \phi_{1}=0$ a solution to the Kodaira-Spencer equation (1). Putting this expansion for $A_{\bar{I}}{ }^{J}$ in (26) we can consider a $Q^{\prime}$ symmetry up to a given order in $t$. In fact, as expected, the nilpotency of $Q^{\prime}$ to a given order, say $n$, corresponds to the Kodaira-Spencer equation at this order (4). When perturbing type B topological matter with an observable of the original theory, i.e., with a closed vector $(0,1)$-form, we remain at first order in Tian's expansion. Taking into account all terms, we obtain the topological theory given by (26) and (32). Notice that the original type B model as well as the model obtained by perturbing to first order can be understood as special cases of the topological theory we have constructed. We will call this theory, in analogy with [19], Kodaira-Spencer topological matter.

One could also wonder if it is possible to construct the perturbed A models by adding the pullback of the Kähler class in the same way that Kodaira-Spencer topological matter has been built, i.e., by adding descendant operators to the unperturbed action. The answer to this question is positive. The action resulting after adding the corresponding two-form descendant to the unperturbed type A action turns out to differ from the one in which just the Kähler class is added in a $Q$-exact term. Since this $Q$-exact term can be disregarded there exist a complete paralelism between perturbed type A and perturbed type B models.

In the rest of the paper we will briefly analyze Kodaira-Spencer topological matter using the standard tools of topological field theories $[1,5,20]$. First notice that the action (31) is $Q^{\prime}$-exact, so we can work in the weak coupling regime as in type B models. Path integrals will be localized in field configurations that can be easily found using Witten's fixed point theorem [5,21]. It suffices to look for the fixed points of the $Q^{\prime}$ fermionic symmetry. According to (26), these correspond to,

$$
\begin{aligned}
\eta^{\bar{I}} & =0, \\
d x^{J}+{A_{\bar{I}}}^{J} d x^{\bar{I}} & =0,
\end{aligned}
$$


where $d$ denotes the exterior differential on $\Sigma$. Notice that the second equation in (33) implies that $d A_{\bar{I}}^{J} \wedge d x^{\bar{I}}=0$, which in turn leads to $\bar{\partial} A=0$. This contradicts Kodaira-Spencer equation, so the only solution to the differential system in (33) is $d x^{I}=d x^{\bar{I}}=0$, and we recover the localization of type B model on constant configurations. Path integrals reduce to integrals over the manifold $M$ and fermionic integrals over the zero modes of $\eta^{\bar{I}}, \theta_{I}$ and $\rho_{\mu}^{I}$ so we have the same constraints on ghost number as in the type B model [5].

Kodaira-Spencer topological matter gives a very different class of observables than ordinary type $\mathrm{B}$ models. This is obviously related to the fact that it describes the moduli space of complex structures by means of the vector $(0,1)$-form $A_{\bar{I}}{ }^{J}$. If one takes a function $f\left(x^{I}, x^{\bar{J}}\right)$, the condition of observable now reads,

$$
\partial_{\bar{K}} f \eta^{\bar{K}}-A_{\bar{K}}{ }^{J} \partial_{J} f \eta^{\bar{K}}=0,
$$

which is just the condition for $f$ to be a holomorphic function in the new complex structure defined by $A_{\bar{I}}^{J}[15]$. One can easily obtain the condition for $V_{\bar{I}}^{J} \eta^{\bar{I}} \theta_{J}$ to be an observable,

$$
\left[Q^{\prime}, V_{\bar{I}}^{J} \eta^{\bar{I}} \theta_{J}\right]=\left(\partial_{\bar{K}} V_{\bar{I}}^{J}-A_{\bar{K}}^{M} \partial_{M} V_{\bar{I}}^{J}-V_{\bar{K}}^{M} \partial_{M} A_{\bar{I}}^{J}\right) \eta^{\bar{K}} \eta^{\bar{I}} \theta_{J}+V_{\bar{I}}^{J} \eta^{\bar{I}} \frac{\delta S^{\prime}}{\delta F^{J}}=0
$$

Modulo field equations, this is equivalent to the following equation for the vector $(0,1)$-form $V$ :

$$
\bar{\partial} V=[A, V]
$$

where the bracket is the same as the one defined in (2). A similar condition is obtained for a general operator of the form (15).

As stated in our brief review on Kodaira-Spencer theory, the vector $(0,1)$-form $A_{\bar{I}}{ }^{J}$ depends on a set of $s$ parameters $t_{1}, \cdots, t_{s}$, where $s$ is the dimension of the moduli space of complex structures. Choosing the Kodaira- Spencer-Kuranishi 
coordinates we have in fact the series expansion $[15,17]$ :

$$
A(t)=\sum_{i=1}^{s} A_{i} t_{i}+\cdots+\sum_{i_{1}+\cdots+i_{s}=n} A_{i_{1} \cdots i_{s}} t_{1}^{i_{1}} \cdots t_{s}^{i_{s}}+\cdots
$$

where the $A_{i}$ are a basis for $H^{1}(M, T M)$ and the $A_{i_{1} \cdots i_{s}}$ are vector $(0,1)$-forms that always exists thanks to Tian's lemma [16]. Although $A_{\bar{I}}^{J} \eta^{\bar{I}} \theta_{J}$ is not itself an observable, the first derivatives of the vector $(0,1)$-form with respect to the parameters $t_{i}$, which we will denote by $\partial_{i} A$, verify (36), as one can see taking the derivative of the Kodaira-Spencer equation (1) with respect to $t_{i}$.

Let us analyze some of the relevant vacuum expectation values when $\Sigma$ is a genus zero Riemann surface and $M$ is a Calabi-Yau threefold. In this case there are $\eta^{\bar{I}}$ and $\theta_{I}$ zero modes but no $\rho_{\mu}^{I}$ zero modes. The ghost number selection rule allows non zero three-point correlation functions for observables of the form $A_{\bar{I}}{ }^{J} \eta^{\bar{I}} \theta_{J}$. After the integration of the constant anticommuting zero modes the path integral reduces to an integral over the manifold $M$. The correlation function is obtained multiplying the antisymmetrized product $\partial_{i} A \wedge \partial_{j} A \wedge \partial_{k} A$ by the square of the holomorphic $(3,0)$-form $\Omega_{0}$ associated to the Calabi-Yau manifold $M$ :

$$
\left\langle\partial_{i} A_{\bar{I}}^{J} \eta^{\bar{I}} \theta_{J} \partial_{j} A_{\bar{K}}{ }^{L} \eta^{\bar{K}} \theta_{L} \partial_{k} A_{\bar{M}}{ }^{N} \eta^{\bar{M}} \theta_{N}\right\rangle=\int_{M}\left[\left(\partial_{i} A \wedge \partial_{j} A \wedge \partial_{k} A\right) \perp \Omega_{0}\right] \wedge \Omega_{0},
$$

where $\perp$ denotes the inner product. Notice that this prescription for the computation of the path integral is defined up to a numerical normalization factor that can be understood as the freedom in the choice of the normalization of the $(3,0)$ form $\Omega_{0}$ [5]. As shown in [19], this are, again up to a factor, the Yukawa couplings $C_{i j k}(t)$, i.e., the Yukawa couplings evaluated at a point of the moduli space of complex structures parametrized by $t$. This follows from Todorov's expression for the deformation of the holomorphic $(3,0)$-form as one changes the complex structure 
$[17]$

$$
\Omega_{t}=\Omega_{0}+A \perp \Omega_{0}-(A \wedge A) \perp \Omega_{0}-(A \wedge A \wedge A) \perp \Omega_{0},
$$

and the following expression for the Yukawa couplings [18, 12]:

$$
C_{i j k}(t)=-\int_{M} \Omega_{t} \wedge \partial_{i} \partial_{j} \partial_{k} \Omega_{t} .
$$

Notice that the mirror computation in [12] involves precisely the Yukawa couplings for a generic point in the space of the complex structures, and compares them with an expansion depending on the parameters of the moduli space of Kähler forms. It has been shown [14] that type A topological matter naturally gives rise to an expansion for the three-point correlation functions which have the polynomial form:

$$
f_{i j k}=N_{i j k}^{0}+N_{a b c}^{1} q_{1}+N_{i j k}^{2} q_{2}+\cdots+N_{i j k}^{\cdots} q_{1}^{2} q_{2}+\cdots
$$

The coefficients $N_{i j k}^{v}$ are intersection numbers of cycles in a moduli space of holomorphic maps from $\Sigma$ to $M$, and the $q_{i}$ are parameters for the Kähler form. Now it seems that it is Kodaira-Spencer topological matter rather than the usual type $\mathrm{B}$ topological matter the one which naturally gives the mirror quantity computed in [12] and contains the information about the moduli space of complex structures as encoded in $A$. Moreover, mirror symmetry between type $\mathrm{A}$ and type $\mathrm{B}$ models seems enhanced when taking Kodaira-Spencer topological matter: if one substitutes (37) in (38), the following expansion is obtained for the Yukawa coupling:

$$
\begin{aligned}
& C_{i j k}(t)=\int_{M}\left[\left(A_{i} \wedge A_{j} \wedge A_{k}\right) \perp \Omega_{0}\right] \wedge \Omega_{0} \\
& +\left(\int_{M}\left[\left(A_{1 i} \wedge A_{j} \wedge A_{k}\right) \perp \Omega_{0}\right] \wedge \Omega_{0}+\int_{M}\left[\left(A_{i} \wedge A_{1 j} \wedge A_{k}\right) \perp \Omega_{0}\right] \wedge \Omega_{0}+\cdots\right) t_{1} \\
& +\cdots
\end{aligned}
$$

In this expansion the coefficients are integer combinations of the various couplings between the vector $(0,1)$-forms which appear in Tian's expansion of the solution 
to the Kodaira-Spencer equation, and the $t_{i}$ are the Kodaira-Spencer-Kuranishi parameters of the complex structure. One can regard (41) and (42) as mirror expansions and exploit this fact once the mirror map relating complex and Kähler parameters is known.

The results presented here give rise to many issues. Of course, it is worth pursuing the study of Kodaira-Spencer topological matter by itself or as an intermediate step to understand the extended moduli space of topological sigma models [5]. This seems to be a promising approach to the mirror symmetry and to the mirror map between moduli spaces from the point of view of topological field theories. But perhaps the most urgent question is the relation of Kodaira-Spencer topological matter with the Kodaira-Spencer theory of gravity presented in [19]. It would be interesting to know if the topological theory we have constructed, which includes the field $A_{\bar{I}} J$ as a background field, gives rise to the six-dimensional action of Kodaira-Spencer theory of gravity. Notice that the third derivatives of this action with respect to the moduli parameters give, at the tree level, the Yukawa couplings [19]. In [22] it was shown in the framework of topological conformal field theories that perturbations of a topological theory can be encoded in a free energy function $F(t)$, so that three-point correlation functions at genus zero are given by $c_{i j k}(t)=\partial_{i} \partial_{j} \partial_{k} F(t)$. In this way the action of the Kodaira-Spencer theory of gravity could be the analog of free energy for Kodaira-Spencer topological matter. We expect to report on this in the future.

Acknowledgements: We would like to thank A. V. Ramallo for very helpful discussions. This work was supported in part by DGICYT under grant PB90-0772 and by CICYT under grants AEN93-0729 and AEN94-0928. 


\section{REFERENCES}

1. E. Witten, Comm. Math. Phys. 118(1988), 411

2. T. Eguchi and S.K. Yang, Mod. Phys. Lett. A5(1990), 1693

3. C. Vafa, Mod. Phys. Lett. A6(1991), 337

4. J.M.F. Labastida and P.M. Llatas, Nucl. Phys. B379(1992), 220

5. E. Witten, "Mirror Manifolds and Topological Field Theory", in Essays on Mirror Manifolds, ed. S.-T. Yau (International Press, 1992)

6. C. Vafa, "Topological Mirrors and Quantum Rings", in Essays on Mirror Manifolds, ed. S.-T. Yau (International Press, 1992)

7. L.J. Dixon, in Superstrings, Unified Theories and Cosmology 1987, (G. Furlan et al., eds.) World Scientific, 1988, pag. 67

8. W. Lerche, C. Vafa and N.P. Warner, Nucl. Phys. B324(1989), 427

9. B.R. Greene and M.R. Plesser, Nucl. Phys. B338(1990), 15

10. P. Candelas, M. Lynker and R. Schmmrigk, Nucl. Phys. B341(1990), 383

11. P.S. Aspinwall, C.A. Lutken and G.G. Ross, Phys. Lett. B241(1990), 373

12. P. Candelas, X.C. de la Ossa, P.S. Green and L. Parkes, Nucl. Phys. B359(1991), 21, Phys. Lett. B258(1991), 118

13. S. Ferrara, M. Bodner and A.C. Cadavid, Phys. Lett. B247(1990), 25

14. P.S. Aspinwall and D.R. Morrison, Comm. Math. Phys. 151(1993), 245

15. K. Kodaira, Complex manifolds and deformation of complex structures, Springer, 1985

16. G. Tian, in S.-T. Yau (ed.), Mathematical Aspects of String Theory, World Scientific,1987; G. Tian, in S.-T. Yau (ed.), Essays on Mirror Manifolds, International Press, 1992

17. A.N. Todorov, Comm. Math. Phys. 126(1989), 325 
18. P. Candelas and X. de la Ossa, Nucl. Phys. B355(1991), 455

19. M. Bershadsky, S. Cecotti, H. Ooguri and C. Vafa, Kodaira-Spencer Theory of Gravity and Exact Results for Quantum String Amplitudes, HUTP-93/A025

20. J.M.F. Labastida and P.M. Llatas, Phys. Lett. B271(1991), 101

21. E. Witten, Nucl. Phys. B371(1992), 191

22. R. Dijkgraaf, H. Verlinde and E. Verlinde, Nucl. Phys. B352(1990), 59 